\title{
Positive solutions for Neumann boundary value problems of nonlinear second-order integro- differential equations in ordered Banach spaces
}

He Yang ${ }^{{ }^{*}}$ and Yue Liang ${ }^{2}$

\author{
* Correspondence: \\ yanghe256@163.com \\ 'Department of Mathematics, \\ Northwest Normal University, \\ Lanzhou 730070, People's Republic \\ of China \\ Full list of author information is \\ available at the end of the article
}

\begin{abstract}
The paper deals with the existence of positive solutions for Neumann boundary value problems of nonlinear second-order integro-differential equations

$$
-u^{\prime \prime}(t)+M u(t)=f(t, u(t),(S u)(t)), 0<t<1, u^{\prime}(0)=u^{\prime}(1)=\theta
$$

and

$$
u^{\prime \prime}(t)+M u(t)=f(t, u(t),(S u)(t)), 0<t<1, u^{\prime}(0)=u^{\prime}(1)=\theta
$$

in an ordered Banach space $E$ with positive cone $K$, where $M>0$ is a constant, $f:[0$, $1] \times K \times K \rightarrow K$ is continuous, $S: C([0,1], K) \rightarrow C([0,1], K)$ is a Fredholm integral operator with positive kernel. Under more general order conditions and measure of noncompactness conditions on the nonlinear term $f$, criteria on existence of positive solutions are obtained. The argument is based on the fixed point index theory of condensing mapping in cones.

Mathematics Subject Classification (2000): 34B15; 34G20.

Keywords: nonlinear second-order integro-differential equation, Neumann boundary value problem, positive solution, condensing mapping, fixed point index theorem
\end{abstract}

\section{Introduction}

Let $E$ be an ordered Banach space, whose positive cone $K$ is normal with a normal constant $N_{0}$, that is, if $\theta \leq x \leq y$, then $\|x\| \leq N_{0}\|y\|$, where $\theta$ is the zero element in $E$. We consider the existence of positive solutions for nonlinear second-order integro-differential equations

$$
-u^{\prime \prime}(t)+M u(t)=f(t, u(t),(S u)(t)), 0<t<1
$$

and

$$
u^{\prime \prime}(t)+M u(t)=f(t, u(t),(S u)(t)), 0<t<1
$$

satisfying Neumann boundary conditions

$$
u^{\prime}(0)=u^{\prime}(1)=\theta,
$$


where $M>0$ is a constant, $f: I \times K \times K \rightarrow K$ is continuous, $I=[0,1]$, and

$$
(S u)(t)=\int_{0}^{1} D(t, s) u(s) \mathrm{d} s
$$

is a Fredholm integral operator with integral kernel $D \in C\left(I \times I, \mathbb{R}^{+}\right)$. For convenience, we denote by $\bar{D}:=\max _{(t, s) \in I \times I} D(t, s)$, and by $\underline{D}:=\min _{(t, s) \in I \times I} D(t, s)$. In the following discussions, we always assume that $\bar{D}>0$.

The existence of positive solutions for ordinary differential equations with certain boundary conditions has been studied by many authors, see [1-5] and the references therein. At first, Guo and Lakshmikantham in [1] discussed the existence of positive solutions for two-point boundary value problem(BVP)

$$
\left\{\begin{array}{l}
-u^{\prime \prime}(t)=f(t, u(t)), t \in I \\
u(0)=u(1)=0
\end{array}\right.
$$

in a Banach space $E$, where $f: I \times K \rightarrow K$ is continuous. By using cone expansion and compression fixed point theorem of condensing mapping, they proved that, if the nonlinear term $f$ satisfies the measure of noncompactness condition

$\left(P_{0}\right)$ For any $R>0, f$ is uniformly continuous on $I \times K_{R}$, and there exists a constant $L \in\left(0, \frac{1}{2}\right)$ such that

$$
\alpha(f(t, B)) \leq L \alpha(B),
$$

for any $t \in I$ and $B \in K_{R}$, where $K_{R}=K \cap \bar{B}(\theta, R)$

and one of the following increasing conditions:

$\left(P_{2}\right) \lim _{x \in K,\|x\| \rightarrow 0} \max _{t \in I} \frac{\|f(t, x)\|}{\|x\|}=0$, and there exist $0<\beta<\gamma<1, \varphi \in K^{*}$ such that $\varphi(x)$ $>0$ for any $x>\theta$ and $\lim _{x \in K,\|x\| \rightarrow+\infty} \min _{\beta \leq t \leq \gamma} \frac{\phi(f(t, x))}{\phi(x)}=+\infty$,

$\left(P_{2}\right)\left(P_{2}\right) \lim _{x \in K,\|x\| \rightarrow+\infty} \max _{t \in I} \frac{\|f(t, x)\|}{\|x\|}=0$, and there exist $0<\beta<\gamma<1, \varphi \in K^{*}$ such that $\varphi(x)>0$ for any $x>\theta$ and $\lim _{x \in K,|| x|| \rightarrow 0} \min _{\beta \leq t \leq \gamma} \frac{\phi(f(t, x))}{\phi(x)}=+\infty$,

then, the $\operatorname{BVP}(4)$ has at least one positive solution. Later, the same technique is employed successfully in [2] in proving the existence of positive solutions for twopoint boundary value problems of ordinary differential equations in $\mathbb{R}$. Recently, this technique is used in [3-5] to investigate the existence of positive solutions for secondorder ordinary differential equations

$$
-u^{\prime \prime}(t)+M u(t)=f(t, u(t)), 0<t<1
$$

and

$$
u^{\prime \prime}(t)+M u(t)=f(t, u(t)), 0<t<1
$$

with Neumann boundary conditions $(3)$ in $\mathbb{R}$. Obviously, the conditions $\left(P_{1}\right)$ and $\left(P_{2}\right)$ are an extension of sup-linear condition

$$
\left(P_{1}\right)^{*} f^{0}:=\lim _{x \rightarrow 0^{+}} \max _{t \in I} \frac{f(t, x)}{x}=0, f_{\infty}:=\lim _{x \rightarrow+\infty} \min _{t \in I} \frac{f(t, x)}{x}=+\infty
$$

and sub-linear condition 
$\left(P_{2}\right)^{*} f_{0}:=\lim _{x \rightarrow 0^{+}} \min _{t \in I} \frac{f(t, x)}{x}=+\infty, f^{\infty}:=\lim _{x \rightarrow+\infty} \max _{t \in I} \frac{f(t, x)}{x}=0$

in [2-4] in $E$. On the other hand, the limits conditions $\left(P_{1}\right)^{*}$ and $\left(P_{2}\right)^{*}$ are equivalent to the inequality conditions $\left(P_{1}\right)^{* * *}$ and $\left(P_{2}\right)^{* *}$ in $\mathbb{R}$ :

$\left(P_{1}\right)^{* * *}$ For any $\varepsilon>0$, there exists $\delta>0$ such that $f(t, x) \leq \varepsilon x$ for any $0 \leq x<\delta$; For any $C>0$, there exists $h \in C^{+}(I)$ such that $f(t, x) \geq C x-h(t)$,

$\left(P_{2}\right)^{* * *}$ For any $C>0$, there exists $\delta>0$ such that $f(t, x) \geq C x$ for any $0 \leq x<\delta$; For any $\varepsilon>0$, there exists $h \in C^{+}(I)$ such that $f(t, x) \leq \varepsilon x+h(t)$.

Clearly, the inequality conditions $\left(P_{1}\right)^{* * *}$ and $\left(P_{2}\right)^{* * *}$ are more convenient to verify and apply in applications than the limits conditions $\left(P_{1}\right)^{*}$ and $\left(P_{2}\right)^{*}$ do.

In this paper, we will improve and extend the results in [1-4]. At first, by applying a new estimate of measure of noncompactness, we will delete the condition that $f$ is uniformly continuous on $I \times K_{R}$ in the assumption $\left(P_{0}\right)$, see the conditions $\left(H_{0}\right)$ or $\left(H_{0}\right)^{*}$. Then, more general order conditions (see conditions $\left(H_{1}\right)$ and $\left(H_{2}\right)$ ) are also presented in this paper to guarantee the existence of positive solutions for the Neumann boundary value problem (1) and (3) or (2) and (3) of nonlinear second-order integro-differential equations. These order conditions are a natural extension of the inequality conditions $\left(P_{1}\right)^{* * *}$ and $\left(P_{2}\right)^{* * *}$ in ordered Banach spaces. The argument of the paper is based on the fixed point index theory of condensing mapping in cones.

\section{Preliminaries}

First, we consider the boundary value problem (1) and (3) with $M>0$.

To obtain a solution of the boundary value problem (1) and (3), we require a mapping whose kernel $G(t, s)$ is the Green's function of the boundary value problem

$$
\left\{\begin{array}{l}
-u^{\prime \prime}(t)+M u(t)=\theta, 0<t<1 \\
u^{\prime}(0)=u^{\prime}(1)=\theta
\end{array}\right.
$$

It is known in [3-5] that

$$
G(t, s)=\left\{\begin{array}{l}
\frac{\cosh (m(1-t)) \cdot \cosh (m s)}{m \sinh m}, 0 \leq s \leq t \leq 1, \\
\frac{\cosh (m(1-s)) \cdot \cosh (m t)}{m \sinh m}, 0 \leq t \leq s \leq 1,
\end{array}\right.
$$

where $m=\sqrt{M}, \cosh x=\frac{\mathrm{e}^{x}+\mathrm{e}^{-x}}{2}, \sinh x=\frac{\mathrm{e}^{x}-\mathrm{e}^{-x}}{2}$. Furthermore, a direct calculation shows that

$$
0<\frac{1}{m \sinh m} \leq G(t, s) \leq \frac{\cosh ^{2} m}{m \sinh m}
$$

and $\int_{0}^{1} G(t, s) \mathrm{d} s=\frac{1}{M}$.

Let $(X,\|\cdot\|)$ be a Banach space, $C(I, X)$ denote the Banach space of all continuous $X$-valued functions on interval $I$ with the norm $\|u\|_{C}=\max \{\|u(t)\|: t \in I\}$. Let $\alpha(\cdot)$ denote the Kuratowski measure of noncompactness of the bounded set in $X$ and $C(I$, $X)$. For the details of definition and properties of the measure of noncompactness, see [6]. For any $B \subset C(I, X)$ and $t \in I$, let $B(t):=\{u(t): u \in B\} \subset X$. If $B$ is bounded in $C(I$, $X)$, then $B(t)$ is bounded in $X$, and $\alpha(B(t)) \leq \alpha(B)$. Some results of $\alpha(\cdot)$ are given in the following lemma. 
Lemma 1 [7-11]Let $\times$ be a Banach space. Then, we have the following results:

(1) If $B \subset C(I, X)$ is a bounded and equicontinuous set, then $\alpha(B(t))$ is continuous on $I$, and

$$
\alpha(B)=\max _{t \in I} \alpha(B(t))=\alpha(B(I)),
$$

(2) If $B \subset X$ is a bounded set, $T: X \rightarrow X$ is a linear bounded operator, then

$$
\alpha(T(B)) \leq\|T\| \cdot \alpha(B),
$$

(3) If $B=\left\{u_{n}\right\} \subset C(I, X)$ is a bounded and countable set, then $\alpha(B(t))$ is Lebesgue integrable on $I$, and

$$
\alpha\left(\left\{\int_{I} u_{n}(t) \mathrm{d} t\right\}\right) \leq 2 \int_{I} \alpha(B(t)) \mathrm{d} t,
$$

(4) If $B \subset X$ is a bounded set, then there exists a countable subset $B_{0} \subset B$ such that

$$
\alpha(B) \leq 2 \alpha\left(B_{0}\right),
$$

(5) If $J=[a, b], u \in C(J, X), \phi \in C\left(J, \mathbb{R}^{+}\right)$, then

$$
\int_{a}^{b} \varphi(s) u(s) \mathrm{d} s \in\left(\int_{a}^{b} \varphi(s) \mathrm{d} s\right) \cdot \overline{C o} U(J),
$$

where $U(J):=\{u(t): t \in \mathcal{H}$.

Let $E$ be an ordered Banach space, whose positive cone $K$ is normal with a normal constant $N_{0}$. We define an operator $Q: C(I, K) \rightarrow C(I, K)$ by

$$
(Q u)(t)=\int_{0}^{1} G(t, s) f(s, u(s),(S u)(s)) \mathrm{d} s, \quad t \in I .
$$

Since $G(t, s)>0$ and $f: I \times K \times K \rightarrow K$ is continuous, $Q: C(I, K) \rightarrow C(I, K)$ is continuous. Obviously, a positive solution of the boundary value problem (1) and (3) is equivalent to a nonzero fixed point of the operator $Q$. Next, we will use fixed point index theorem of condensing mapping in cone to seek the nonzero fixed point of $Q$. For this purpose, we first prove that $Q: C(I, K) \rightarrow C(I, K)$ is a condensing mapping. A mapping $Q: C(I, K) \rightarrow C(I, K)$ is said to be a condensing mapping if for any bounded set $B \subset C(I, K)$, we have $\alpha(Q(B))<\alpha(B)$.

Lemma 2 Assume that $f \in C(I \times K \times K, K)$ satisfies the following condition $\left(H_{0}\right)$ For any $R>0, f\left(I \times K_{R} \times K_{R}\right)$ is bounded, and there exist two constants $L_{1}, L_{2}>0$ with $L_{1}+L_{2} \bar{D}<\frac{M}{4}$ such that

$$
\alpha\left(f\left(t, B_{1}, B_{2}\right)\right) \leq L_{1} \alpha\left(B_{1}\right)+L_{2} \alpha\left(B_{2}\right),
$$

for any $t \in I$ and $B_{1}, B_{2} \subset K_{R}$, where $K_{R}$ is defined as in condition $\left(P_{0}\right)$.

Then $Q: C(I, K) \rightarrow C(I, K)$ defined by (8) is a condensing mapping.

Proof. From (8) and assumption $\left(H_{0}\right)$, it follows that $Q$ maps bounded sets of $C(I, K)$ into bounded and equicontinuous sets. Let $B \subset C(I, K)$ be a bounded set, we show that $\alpha(Q(B))<\alpha(B)$. Let $R:=\sup \left\{\|u\|_{C}+\|S u\|_{C}: u \in B\right\}$, then for any $t \in I$, we have $B(t)$ 
$\subset K_{R},(S B)(t) \subset K_{R}$. From Lemma 1(4), there exists a countable subset $B_{0}=\left\{u_{n}\right\} \subset B$ such that $\alpha(Q(B)) \leq 2 \alpha\left(Q\left(B_{0}\right)\right)$. For any $t \in I$, from Lemma 1(3) and assumption $\left(H_{0}\right)$, we have

$$
\begin{aligned}
\alpha\left(Q\left(B_{0}\right)(t)\right) & =\alpha\left(\left\{\int_{0}^{1} G(t, s) f\left(s, u_{n}(s),\left(S u_{n}\right)(s)\right) \mathrm{d} s: n \in \mathbb{N}\right\}\right) \\
& \leq 2 \int_{0}^{1} G(t, s) \alpha\left(\left\{f\left(s, u_{n}(s),\left(S u_{n}\right)(s)\right): n \in \mathbb{N}\right\}\right) \mathrm{d} s \\
& =2 \int_{0}^{1} G(t, s) \alpha\left(f\left(s, B_{0}(s),\left(S B_{0}\right)(s)\right)\right) \mathrm{d} s \\
& \leq 2 \int_{0}^{1} G(t, s)\left(L_{1} \alpha\left(B_{0}(s)\right)+L_{2} \alpha\left(\left(S B_{0}\right)(s)\right)\right) \mathrm{d} s .
\end{aligned}
$$

Since $S B_{0}$ is bounded and equicontinuous, by Lemma 1(1) and Lemma 1(2), we have

$$
\alpha\left(\left(S B_{0}\right)(s)\right) \leq \max _{s \in I} \alpha\left(\left(S B_{0}\right)(s)\right)=\alpha\left(S B_{0}\right) \leq\|S\| \cdot \alpha\left(B_{0}\right) \leq \bar{D} \cdot \alpha\left(B_{0}\right) .
$$

Hence, by the properties of measure of noncompactness, we have

$$
\alpha\left(Q\left(B_{0}\right)(t)\right) \leq 2\left(L_{1}+L_{2} \bar{D}\right) \int_{0}^{1} G(t, s) \mathrm{d} s \cdot \alpha\left(B_{0}\right) \leq \frac{2\left(L_{1}+L_{2} \bar{D}\right)}{M} \alpha(B) .
$$

Since $Q\left(B_{0}\right)$ is bounded and equicontinuous, from Lemma 1(1) and assumption $\left(H_{0}\right)$, we have

$$
\alpha(Q(B)) \leq 2 \alpha\left(Q\left(B_{0}\right)\right)=2 \max _{t \in I} \alpha\left(Q\left(B_{0}\right)(t)\right) \leq \frac{4\left(L_{1}+L_{2} \bar{D}\right)}{M} \alpha(B)<\alpha(B) .
$$

This implies that $Q: C(I, K) \rightarrow C(I, K)$ is a condensing mapping. The proof is completed. $\square$

Remark 1 Comparing with assumption $\left(P_{0}\right)$, assumption $\left(H_{0}\right)$ does not require that $f$ is uniformly continuous on $I \times K_{R}$. Hence assumption $\left(H_{0}\right)$ is weaker than assumption $\left(P_{0}\right)$.

Define a cone in $C(I, K)$ by

$$
P:=\{u \in C(I, K): u(t) \geq \sigma u(\tau), \quad \forall t, \tau \in I\},
$$

where $\sigma=\frac{1}{\cosh ^{2} m}$. Before starting our main results, we give the following lemma.

Lemma $3 Q(C(I, K)) \subset P$.

Proof. For any $u \in C(I, K)$, from (7) and (8), for any $\tau \in I$, we have

$$
(\mathrm{Qu})(\tau) \leq \frac{\cosh ^{2} m}{m \sinh m} \int_{0}^{1} f(s, u(s),(S u)(s)) \mathrm{d} s .
$$


On the other hand, for any $t \in I$, we have

$$
(Q u)(t) \geq \frac{1}{m \sinh m} \int_{0}^{1} f(s, u(s),(S u)(s)) \mathrm{d} s \geq \sigma(Q u)(\tau),
$$

i. e. $Q u \in P$. This implies that $Q(C(I, K)) \subset P$. The proof is completed.

In order to use fixed point index theorem of condensing mapping in cones to seek nonzero fixed point of $Q$, we also need the following lemmas.

Lemma 4 [12] Let $\times$ be a Banach space, $P \subset X$ be a cone, $\Omega \subset X$ be a bounded open set, $\theta \in \Omega, A: P \cap \bar{\Omega} \rightarrow P$ be a condensing mapping. If $u \neq \mu A u$ for any $u \in \partial \Omega \cap P$ and $0<\mu \leq 1$, then $i(A, P \cap \Omega, P)=1$.

Lemma 5 [13] Let $\times$ be a Banach space, $P \subset X$ be a cone, $\Omega \subset X$ be a bounded open set, $A: P \cap \bar{\Omega} \rightarrow P$ be a condensing mapping. If there exists $a v_{0} \in P \backslash\{\theta\}$ such that $u$ $A u \neq \tau v_{0}$ for any $u \in \partial \Omega \cap P$ and $\tau \geq 0$, then $i(A, P \cap \Omega, P)=0$.

\section{Main results}

For convenience, for any $r>0$, let $P_{r}:=\left\{u \in P:\|u\|_{C}<r\right\}$. Then, $\partial P_{r}=\left\{u \in P:\|u\|_{C}\right.$ $=r$. Now, we are in the position to state and prove our main results.

Theorem 1 Let $E$ be an ordered Banach space, whose positive cone $K$ is normal. If $M$ $>0$ and $f \in C(I \times K \times K, K)$ satisfies the assumption $\left(H_{0}\right)$ and one of the following conditions

$\left(H_{1}\right)$ (1) There exist two constants $a, b>0$ with $a+b \bar{D}<M$ and $\delta>0$ such that

$$
f(t, u, v) \leq a u+b v,
$$

for any $t \in I$ and $u, v \in K_{\delta}$, where $K_{\delta}=K \cap \bar{B}(\theta, \delta)$,

(2) There exist two constants $c, d>0$ with $c+\mathrm{d} \underline{D}>M$ and $h_{0} \in C(I, K)$ such that

$$
f(t, u, v) \geq c u+d v-h_{0}(t)
$$

for any $t \in I$ and $u, v \in K$,

$\left(H_{2}\right)$ (1) There exist two constants $c, d>0$ with $c+\mathrm{d} \underline{D}>M$ and $\delta>0$ such that

$$
f(t, u, v) \geq c u+d v,
$$

for any $t \in I$ and $u, v \in K_{\delta}$,

(2) There exist two constants $a, b>0$ with $a+b \bar{D}<M$ and $h_{0} \in C(I, K)$ such that

$$
f(t, u, v) \leq a u+b v+h_{0}(t)
$$

for any $t \in I$ and $u, v \in K$,

then the boundary value problem (1) and (3) has at least one positive solution.

Proof. Since $f \in C(I \times K \times K, K)$ satisfies assumption $\left(H_{0}\right)$, from Lemmas 2 and 3, we know that $Q: P \rightarrow P$ is a condensing mapping. Next, we will show that the opertor $Q$ defined by $(8)$ has at least one nonzero fixed point when $f$ satisfies assumption $\left(H_{1}\right)$ or $\left(H_{2}\right)$.

If $\left(H_{1}\right)$ holds, let $0<r<\min \left\{\delta, \frac{\delta}{\bar{D}}\right\}$, then for any $t \in I$ and $u \in \partial P_{r}$, we have $\|u(t)\|$ $\leq\|u\|_{C}=r<\delta,\|(S u)(t)\| \leq\|S u\|_{C} \leq \bar{D}\|u\|_{C}=\bar{D} r<\delta$. Hence from assumption $\left(H_{1}\right)(1)$, we have 


$$
f(t, u(t),(S u)(t)) \leq a u(t)+b(S u)(t)
$$

We now prove that $u \neq \mu Q u$ for any $u \in \partial P_{r}$ and $0<\mu \leq 1$. In fact, if there exist $u_{0}$ $\in \partial P_{r}$ and $0<\mu_{0} \leq 1$ such that $u_{0}=\mu_{0} Q u_{0}$, then by the definition of operator $Q, u_{0}(t)$ satisfies the equation

$$
-u_{0}^{\prime \prime}(t)+M u_{0}(t)=\mu_{0} f\left(t, u_{0}(t),\left(S u_{0}\right)(t)\right), 0<t<1
$$

and Neumann boundary condition (3). Integrating on both sides of Equation (10) from 0 to 1 , by (9), we have

$$
\begin{aligned}
M \int_{0}^{1} u_{0}(t) \mathrm{d} t & \leq \int_{0}^{1} f\left(t, u_{0}(t),\left(S u_{0}\right)(t)\right) \mathrm{d} t \leq \int_{0}^{1}\left(a u_{0}(t)+b\left(S u_{0}\right)(t)\right) \mathrm{d} t \\
& \leq(a+b \bar{D}) \int_{0}^{1} u_{0}(t) \mathrm{d} t
\end{aligned}
$$

Combining this inequality with, $a+b \bar{D}<M$, it follows that $\int_{0}^{1} u_{0}(t) \mathrm{d} t \leq \theta$. But from $u_{0} \in C(I, K)$, we have that $u_{0}(t) \geq \theta$ for any $t \in I$, and from $u_{0} \in \partial P_{r}$, we have that $\left\|u_{0}\right\|_{C}=r$. Thus, $u_{0}(t) \geq \theta$ and $u_{0}(t) \otimes \theta$. Therefore, $\int_{0}^{1} u_{0}(t) \mathrm{d} t>\theta$. This is a contradiction. Hence $Q$ satisfies the hypotheses of Lemma 4 in $P_{r}$. From Lemma 4, we have

$$
i\left(Q, P_{r}, P\right)=1 .
$$

On the other hand, let $v_{0} \equiv e$, where $e \in K$ and $\|e\|=1$, then $v_{0}$ is a solution of the boundary value problem (1) and (3) when $f(t, u, S u)=M e$. This implies that $v_{0} \in P$. Next, we show that if $R>0$ large enough, then $u-Q u \neq \tau v_{0}$ for any $u \in \partial P_{R}$ and $\tau \geq 0$. In fact, if there exist $u_{0} \in \partial P_{R}$ and $\tau_{0} \geq 0$ such that $u_{0}-Q u_{0}=\tau_{0} v_{0}$, then by the definition of operator $Q, u_{0}(t)$ satisfies the equation

$$
-u_{0}^{\prime \prime}(t)+M u_{0}(t)=f\left(t, u_{0}(t),\left(S u_{0}\right)(t)\right)+M \tau_{0} v_{0}, 0<t<1
$$

and Neumann boundary condition (3). Integrating on both sides of Equation (12) from 0 to 1 , by assumption $\left(H_{1}\right)(2)$, we have

$$
\begin{aligned}
M \int_{0}^{1} u_{0}(t) \mathrm{d} t & =\int_{0}^{1} f\left(t, u_{0}(t),\left(S u_{0}\right)(t)\right) \mathrm{d} t+M \tau_{0} v_{0} \\
& \geq \int_{0}^{1}\left(c u_{0}(t)+d\left(S u_{0}\right)(t)-h_{0}(t)\right) \mathrm{d} t \\
& \geq(c+\mathrm{d} \underline{D}) \int_{0}^{1} u_{0}(t) \mathrm{d} t-\int_{0}^{1} h_{0}(t) \mathrm{d} t .
\end{aligned}
$$


Consequently, we obtain that

$$
\int_{0}^{1} u_{0}(t) \mathrm{d} t \leq \frac{1}{c+\mathrm{d} \underline{D}-M} \int_{0}^{1} h_{0}(t) \mathrm{d} t .
$$

On the other hand, from $u_{0} \in P$, we have

$$
\int_{0}^{1} u_{0}(t) \mathrm{d} t \geq \int_{0}^{1} \sigma u_{0}(\tau) \mathrm{d} t=\sigma u_{0}(\tau) \geq \theta .
$$

Combining this inequality with (13), and by the normality of cone $K$ in $E$, we obtain that

$$
\left\|u_{0}\right\|_{C} \leq \frac{N_{0}\left\|h_{0}\right\|_{C}}{\sigma(c+\mathrm{d} \underline{D}-M)} \triangleq \bar{R}
$$

Let $R>\max \left\{\delta, \frac{\delta}{\bar{D}}, \bar{R}\right\}$. Then, for any $u \in \partial P_{R}$ and $\tau \geq 0, u-Q u \neq \tau v_{0}$. Hence hypotheses of Lemma 5 hold. By Lemma 5 , we have

$$
i\left(Q, P_{R}, P\right)=0 .
$$

Now, by the additivity of fixed point index, (11) and (15), we have

$$
i\left(Q, P_{R} \backslash \bar{P}_{r}, P\right)=i\left(Q, P_{R}, P\right)-i\left(Q, P_{r}, P\right)=-1 .
$$

Therefore, $Q$ has a fixed point $u^{*}$ in $P_{R} \backslash \bar{P}_{r}$, which satisfies $u^{*}(t) \geq \sigma u^{*}(\tau) \geq \theta$ for any $t, \quad \in \quad I$. By the normality of cone $K$ in $E$, we see that $\left\|u^{*}(t)\right\| \geq \frac{\sigma}{N_{0}}\left\|u^{*}(\tau)\right\|>\frac{\sigma r}{N_{0}}>0$, which implies that $u^{*}$ is a positive solution of the boundary value problem (1) and (3).

Next, we suppose that $\left(H_{2}\right)$ holds. Let $0<r<\min \left\{\delta, \frac{\delta}{\bar{D}}\right\}$, then for any $t \in I$ and $u \in$ $\partial P_{r}$, we have

$$
f(t, u(t),(S u)(t)) \geq c u(t)+d(S u)(t) .
$$

Let $v_{0} \equiv e$, where $e \in K$ and $\|e\|=1$. We now prove that $u$ - Qu $\neq \tau v_{0}$ for any $u \in$ $\partial P_{r}$ and $\tau \geq 0$. In fact, if there exist $u_{0} \in \partial P_{r}$ and $\tau_{0} \geq 0$ such that $u_{0}-Q u_{0}=\tau_{0} v_{0}$, then $u_{0}(t)$ satisfies Equation (12) and Neumann boundary condition (3). From (12) and (16), it follows that

$$
(c+\mathrm{d} \underline{D}-M) \int_{0}^{1} u_{0}(t) \mathrm{d} t \leq-M \tau_{0} v_{0} \leq \theta .
$$

Since $\int_{0}^{1} u_{0}(t) \mathrm{d} t>\theta$, we see that $c+\mathrm{d} \underline{D} \leq M$, which is a contradiction. Hence by Lemma 5, we have

$$
i\left(Q, P_{r}, P\right)=0 .
$$

On the other hand, we show that if $R>0$ large enough, then $u \neq \mu Q u$ for any $u \in$ $\partial P_{R}$ and $0<\mu \leq 1$. In fact, if there exist $u_{0} \in \partial P_{R}$ and $0<\mu_{0} \leq 1$ such that $u_{0}=$ 
$\mu_{0} Q u_{0}$, then $u_{0}(t)$ satisfies Equation (10) and Neumann boundary condition (3). From (10) and assumption $\left(H_{2}\right)(2)$, it follows that

$$
M \int_{0}^{1} u_{0}(t) \mathrm{d} t \leq(a+b \bar{D}) \int_{0}^{1} u_{0}(t) \mathrm{d} t+\int_{0}^{1} h_{0}(t) \mathrm{d} t .
$$

By the proof of (14), we see that $\left\|u_{0}\right\|_{C} \leq \frac{N_{0}\left\|h_{0}\right\|_{C}}{\sigma(M-a-b \bar{D})} \triangleq \bar{R}$. Let $R>\max \left\{\delta, \frac{\delta}{\bar{D}}, \bar{R}\right\}$, then $u \neq \mu Q u$ for any $u \in \partial P_{R}$ and $0<\mu \leq 1$. therefore, by Lemma 4 , we have

$$
i\left(Q, P_{R}, P\right)=1 \text {. }
$$

From (17) and (18), it follows that

$$
i\left(Q, P_{R} \backslash \bar{P}_{r}, P\right)=i\left(Q, P_{R}, P\right)-i\left(Q, P_{r}, P\right)=1 .
$$

Therefore, $Q$ has a fixed point $u^{*}$ in $P_{R} \backslash \bar{P}_{r}$, which is the positive solution of the boundary value problem (1) and (3). The proof is completed.

Theorem 2 Let $E$ be an ordered Banach space, whose positive cone $K$ is normal. If $M$ $>0$ and $f \in C(I \times K \times K, K)$ satisfies the assumption

$\left(H_{0}\right)^{*}$ For any $R>0, f\left(I \times K_{R} \times K_{R}\right)$ is bounded, and there exist two constants $L_{1}, L_{2}$ $>0$ with $2 L_{1}+L_{2} \bar{D}<M$ such that

$$
\alpha\left(f\left(I \times B_{1} \times B_{2}\right)\right) \leq L_{1} \alpha\left(B_{1}\right)+L_{2} \alpha\left(B_{2}\right),
$$

for any $B_{1}, B_{2} \subset K_{R}$, where $K_{R}$ is defined as in $\left(P_{0}\right)$ and the condition $\left(H_{1}\right)$ or $\left(H_{2}\right)$, then the boundary value problem (1) and (3) has at least one positive solution.

Proof. We only need to prove that $Q: C(I, K) \rightarrow C(I, K)$ is a condensing mapping. For any bounded set $B \subset C(I, K)$, let $R:=\sup \left\{\|u\|_{C}+\|S u\|_{C}: u \in B\right\}$, then $B(t) \subset K_{R}$, $(S B)(t) \subset K_{R}$ for any $t \in I$. For any $u \in B$ and $t \in I$, from Lemma 1(5), we have

$$
\begin{aligned}
(Q u)(t) & =\int_{0}^{1} G(t, s) f(s, u(s),(S u)(s)) \mathrm{d} s \\
& \in\left(\int_{0}^{1} G(t, s) \mathrm{d} s\right) \cdot \overline{C o}(\{f(s, u(s),(S u)(s)): s \in I\}) \\
& \subset\left(\int_{0}^{1} G(t, s) \mathrm{d} s\right) \cdot \overline{C o}(f(I \times B(I) \times(S B)(I))) .
\end{aligned}
$$

Consequently, we obtain that

$$
Q(B)(t) \subset\left(\int_{0}^{1} G(t, s) \mathrm{d} s\right) \cdot \overline{C o}(f(I \times B(I) \times(S B)(I))) .
$$

From the properties of measure of noncompactness, Lemma 1(2) and assumption $\left(H_{0}\right)^{*}$, we have 


$$
\begin{aligned}
\alpha(Q(B)(t)) & \leq\left(\int_{0}^{1} G(t, s) \mathrm{d} s\right) \cdot \alpha(f(I \times B(I) \times(S B)(I))) \\
& \leq \frac{1}{M}\left(L_{1} \alpha(B(I))+L_{2} \alpha((S B)(I))\right) \\
& \leq \frac{2 L_{1}+L_{2} \bar{D}}{M} \alpha(B) .
\end{aligned}
$$

From Lemma 1(1) and assumption $\left(H_{0}\right)^{*}$, we have

$$
\alpha(Q(B))=\max _{t \in I} \alpha(Q(B)(t)) \leq \frac{2 L_{1}+L_{2} \bar{D}}{M} \alpha(B)<\alpha(B) .
$$

This implies that $Q: C(I, K) \rightarrow C(I, K)$ is a condensing mapping. The proof is completed.

Remark 2 In assumption $\left(H_{0}\right)^{*}$, we replace $L_{1}+L_{2} \bar{D}<\frac{M}{4}$ by $2 L_{1}+L_{2} \bar{D}<M$, but the condition $\alpha\left(f\left(I \times B_{1} \times B_{2}\right)\right) \leq L_{1} \alpha\left(B_{1}\right)+L_{2} \alpha\left(B_{2}\right)$ is stronger than condition $\alpha\left(f\left(t, B_{1}\right.\right.$, $\left.\left.B_{2}\right)\right) \leq L_{1} \alpha\left(B_{1}\right)+L_{2} \alpha\left(B_{2}\right)(t \in I)$ in $\left(H_{0}\right)$, where $B_{1}, B_{2} \subset K_{R}$. Hence the assumption $\left(H_{0}\right)^{* *}$ is different from assumption $\left(H_{0}\right)$. It is another improvement of assumption $\left(P_{0}\right)$.

The same technique can be carried over for the boundary value problem defined by (2) and (3) with $M \in\left(0, \frac{\pi^{2}}{4}\right)$. We only need to change the Green's function to

$$
G(t, s)=\left\{\begin{array}{l}
\frac{\cos (m(1-t)) \cdot \cos (m s)}{m \sin m}, 0 \leq s \leq t \leq 1, \\
\frac{\cos (m(1-s)) \cdot \cos (m t)}{m \sin m}, 0 \leq t \leq s \leq 1,
\end{array}\right.
$$

and the cone in $C(I, K)$ to

$$
P:=\{u \in C(I, K): u(t) \geq \sigma u(\tau), \quad \forall t, \tau \in I\},
$$

where now $\sigma=\cos ^{2} m$. Similar to the proof of Theorems 1 and 2, we can obtain the following result.

Theorem 3 Let $E$ be an ordered Banach space, whose positive cone $K$ is normal. If < $M<\frac{\pi^{2}}{4}$ and $f 2 C(I \times K \times K, K)$ satisfy the assumption $\left(H_{0}\right)$ or $\left(H_{0}\right) *$ and the condition $\left(H_{1}\right)$ or $\left(H_{2}\right)$, then the boundary value problem defined by (2) and (3) has at least one positive solution.

Remark 3 The conditions $\left(H_{1}\right)$ and $\left(H_{2}\right)$ are a natural extension of the inequality conditions $\left(P_{1}\right)^{* * *}$ and $\left(P_{2}\right)^{* * *}$ in ordered Banach space E. Hence, if $f(t, u, v)=f(t, u)$, then Theorems 1, 2, and 3 improve and extend the main results in [2-4].

Acknowledgements

Research supported by NNSF of China (10871160), the NSF of Gansu Province (0710RJZA103), and Project of NWNUKJCXGC-3-47.

\section{Author details}

${ }^{1}$ Department of Mathematics, Northwest Normal University, Lanzhou 730070, People's Republic of China ${ }^{2}$ Science

College of Gansu Agricultural University, Lanzhou 730070, People's Republic of China

\section{Authors' contributions}

HY carried out the study of the existence of positive solutions of second-order Neumann boundary value problems in ordered Banach spaces, participated in the proof of the main results and drafted the manuscript. YL conceived of the study, participated in the design of the proof. All authors read and approved the final manuscript. 
The authors declare that they have no competing interests.

Received: 8 November 2010 Accepted: 30 September 2011 Published: 30 September 2011

\section{References}

1. Guo, DJ, Lakshmikantham, V: Multiple solutions of two-point boundary value problems of ordinary differential equations in Banach spaces. J Math Anal Appl. 129(1):211-222 (1988). doi:10.1016/0022-247X(88)90243-0

2. Erbe, LH, Wang, HY: On the existence of the positive solutions of ordinary differential equations. Proc Amer Math Soc. 120(3):743-748 (1994). doi:10.1090/S0002-9939-1994-1204373-9

3. Jiang, DQ, Liu, HZ: Existence of positive solutions to second order Neumann boundary value problems. J Math Res Expo. 20(3):360-364 (2000)

4. Sun, JP, Li, WT: Multiple positive solutions to second-order Neumann boundary value problems. Appl Math Comput. 146(1):187-194 (2003). doi:10.1016/50096-3003(02)00535-0

5. Sun, JP, Li, WT, Cheng, SS: Three positive solutions for second-order Neumann boundary value problems. Appl Math Lett. 17, 1079-1084 (2004). doi:10.1016/j.aml.2004.07.012

6. Deimling, K: Nonlinear Functional Analysis. Springer, New York (1985)

7. Guo, DJ, Sun, JX: Ordinary Differential Equations in Abstract Spaces. Shandong Science and Technology Press, Jinan (in Chinese) (2003)

8. Li, YX: The global solutions of initial value problem for abstract semilinear evolution equations. Acta Anal Func Appl 3(4):339-347 (2001). (in Chinese)

9. Heinz, HR: On the behaviour of measure of noncompactness with respect to differentiation and integration of vectorvalued functions. Nonlinear Anal. 7, 1351-1371 (1983). doi:10.1016/0362-546X(83)90006-8

10. Li, YX: Existence of solutions of initial value problems for abstract semilinear evolution equations. Acta Math Sinica 48(6), 1089-1094 (2005). (in Chinese)

11. Li, YX, Wang, X: Positive periodic solutions for ordinary differential equations in ordered Banach spaces. J Northwest Norm University Nat Sci 38(1):1-5 (2002). (in Chinese)

12. Guo, DJ: Nonlinear Functional Analysis. Shandong Science and Technology Press, Jinan (in Chinese) (1985)

13. Yu, QY: Condensing mapping and positive fixed point in partial ordered Banach spaces. J Lanzhou University Nat Sci 2, 23-32 (1979). (in Chinese)

doi:10.1186/1029-242X-2011-73

Cite this article as: Yang and Liang: Positive solutions for Neumann boundary value problems of nonlinear

second-order integro-differential equations in ordered Banach spaces. Journal of Inequalities and Applications 2011 2011:73.

\section{Submit your manuscript to a SpringerOpen ${ }^{\odot}$ journal and benefit from:}

- Convenient online submission

- Rigorous peer review

- Immediate publication on acceptance

- Open access: articles freely available online

- High visibility within the field

- Retaining the copyright to your article

Submit your next manuscript at $\boldsymbol{\wedge}$ springeropen.com 